\title{
Structure, optical and thermal decomposition characters of LDPE graft copolymers synthesized by gamma irradiation
}

\author{
M MADANI \\ Radiation Physics Department, National Centre for Radiation Research and Technology, Nasr City, Cairo, Egypt
}

MS received 9 November 2008; revised 18 April 2009

\begin{abstract}
Methyl methacrylate (MMA) monomer was grafted onto low density polyethylene by the direct method of radiation grafting. The effect of cohesive energy density of different organic solvents on the degree of grafting was investigated. It was found that the extent of grafting depends largely on the kind of solvent, in which the highest degree of grafting was achieved in the presence of dioxane, whereas the lowest degree of grafting occurred in the presence of methanol. This behaviour was attributed to the solubility parameters of the solvent, monomer and polymer. The change in structure of the LDPE graft copolymer films was characterized by scanning electron microscopy, X-ray diffraction, UV/vis absorption and thermogravimetric analysis. The X-ray diffraction results showed a decrease in the crystallinity of LDPE graft copolymer matrix at high degree of grafting. Studies were made on the UV-absorption edge, and indirect allowed transitions with their optical energy gaps are determined. At the same time the Urbach energy was evaluated. The activation energy of the thermal decomposition was calculated according to Horowitz and Metzger method.
\end{abstract}

Keywords. Gamma irradiation; graft copolymerization; spectroscopic analysis; XRD; kinetics of thermal decomposition; activation energy.

\section{Introduction}

Nowadays, the modification of polymers covers radiation cross-linking, radiation induced polymerization (graft polymerization and curing) and the degradation of polymers. Among these, are the modified mechanical, thermal, electrical, and optical properties to fulfill the required characteristics for such goods as packaging and optical devices (Jastrzebski 1977; Utracki 1990; Saq'an et al 2001; Chmielewski et al 2005). Radiation processing can modify the molecular weight, hydrophilicity and all properties of the polymer either by direct irradiation or by radiation grafting with suitable polymeric segments without using any toxic initiator.

The radiation-induced grafting method is the most effective for the introduction of the graft polymer chains, because the molecular chains can propagate through radical species that are generated inside and outside of the film by irradiation (Chen et al 2005). By controlling the experimental conditions (monomer concentration, gamma radiation dose-rate and irradiation environment), one can tailor the final properties of the samples in order to produce new materials for specific applications. Synthetic polymeric membranes continue to be the object of intensive research in the field of polymer, physical and chemical

(madany_2000@yahoo.com) sciences (Billmeyer 1984; Benson 2002; Ferreira et al 2005). This is due to their important role in engineering and technology (Rajindar 1998). Thus radiation-induced grafting is a versatile technique to modify the properties of polymeric membranes.

Low density polyethylene (LDPE) is most commonly used as plastic and has been extensively used as a backbone for radiation grafting with different monomers. This is essentially due to its excellent chemical resistance and high impact strength (Ferreira et al 2005). Due to its high chemical inertness against solvents, acids and bases, polyethylene (PE) as a matrix material became a very popular membrane after grafting with various hydrophilic monomers. Furthermore, LDPE can be used in a variety of forms such as fibres, films, membranes, foams and powders.

Evaluation of the thermal stability and physical properties of modified polymeric materials such as grain size and degree of crystallinity is very important. The X-ray diffraction (XRD) profile is a useful tool for the characterization of crystalline and partially crystalline solids. It is generally known that transmission electron microscope (TEM) can provide a direct image of the grain size distribution. However, TEM imaging is localized within a small region of the sample and the preparation procedures of TEM samples are tedious. Further, grain overlap poses a serious problem. The advantages of XRD over TEM for grain size measurement are obvious, i.e. average grain 
size is determined from larger area of sample and ease of sample preparation. Several theoretical models have been developed to obtain structural information through mathematical analysis of the XRD profile (Warren and Averbach 1950; Klug 1974). The applicability of the XRD line profile analysis in determining the grain size and microstrain in nanocrystalline materials has been carried out extensively by many researchers (Warren and Averbach 1950; Klug and Alexander 1974; Malow and Koch 1997; Inami et al 1999; Jiang et al 1999; Tian and Atzmon 1999; Zhao et al 2001; Qian et al 2002; Ungar 2003). Scanning electron microscope (SEM) studies can yield information on the morphology and topography of graft copolymer systems (Adem et al 2005).

In this work, radiation-induced graft copolymerization of methyl methacrylate (MMA) monomer onto LDPE films in the presence of different organic solvents was investigated. The main aim was to obtain final graft copolymers with high degree of grafting with homogeneous distribution. The characterization and properties of the prepared graft copolymer were studied by employing $\mathrm{X}$-ray diffraction, thermogravimetric analysis (TGA), and scanning electron microscopy (SEM). Also, the study included measurements of optical spectra and determination of the optical energy gaps of these materials as a function of degree of grafting. The optical absorption method provides information regarding the optically induced transitions and the variation in energy bandgap after irradiation. The polymer irradiation leads to a shift in the optical absorption edges, which indicates a lowering of the energy gap. Analysis of optical absorption spectra is one of the most effective tools for understanding and developing the energy band diagram in both crystalline and amorphous materials.

\section{Experimental}

\subsection{Materials}

Low density polyethylene (LDPE) films used throughout this work (thickness $\sim 50 \mu \mathrm{m}$ ) were provided by El-Nasr Co. for Medical Supplies, Cairo, Egypt. Methyl methacrylate monomer (MMA), of purity 99\% pure (Merck, Germany) was used as received. Solvents and chemical reagents were of laboratory grade and were used without further purification.

\subsection{Graft copolymerization onto LDPE}

Strips of low density polyethylene (LDPE) were washed with acetone, dried at $50^{\circ} \mathrm{C}$ in vacuum oven, weighed and then immersed in the monomer/solvent mixtures 50/50 wt \% in glass tubes. The direct radiation grafting method was used as a technique. The glass tubes were then subjected to ${ }^{60} \mathrm{Co}-\gamma$-rays to different irradiation doses at a dose rate of $9.23 \mathrm{kGy} / \mathrm{h}$. Irradiation was carried out in the ${ }^{60} \mathrm{Co}$ gamma source (model GB150 type B) manufactured by the AEA of Canada and located at NCRRT, Egypt. The grafted films were removed and washed thoroughly with acetone to remove the residual monomer and the homopolymer which may be accumulated on the film. The films were dried in vacuum oven at $50-60^{\circ} \mathrm{C}$ for $24 \mathrm{~h}$ and weighed. The degree of grafting was determined as follows

Degree of grafting $(\%)=\left[\left(W_{\mathrm{g}}-W_{\mathrm{o}}\right) / W_{\mathrm{o}}\right] \times 100$,

where $W_{\mathrm{o}}$ and $W_{\mathrm{g}}$ represent the weight of the ungrafted and grafted films, respectively.

\subsection{Structural characterization and thermal decomposition}

The fracture surface of polymeric films was observed by scanning electron microscopy (SEM) (Jeol, JSM) at a voltage of $30 \mathrm{kV}$. The fracture surfaces were pre-coated with a thin gold layer to reduce charging in the SEM. $X$-ray diffraction (XRD) patterns were recorded using Shimadzu diffractometer XD-D1 X-ray diffraction spectrometer with a copper target at an operating voltage of $40 \mathrm{kV}$ and an electric current of $30 \mathrm{~mA}$. The optical absorption measurements in the wavelength range from 190 to $900 \mathrm{~nm}$ were carried out using spectrophotometer model UV-VIS Milton Roy Spectronic 1201. The observed optical energy gaps and the energy gap tails were determined from the measured absorption spectra. Thermogravimetric analysis (TGA) was performed using Shimadzu50 instrument (Japan) at a heating rate of $10^{\circ} \mathrm{C} / \mathrm{min}$ under flowing nitrogen $(20 \mathrm{ml} / \mathrm{min})$ from ambient temperature to $500^{\circ} \mathrm{C}$. The primary TGA thermograms were used to determine the kinetic parameters such as activation energy and order of the thermal decomposition reaction.

\section{Results and discussion}

\subsection{Effect of irradiation dose on grafting yield}

The nature of polymer backbone and the reactivity of monomers are important in radiation grafting. The reactivity of monomers depends upon different factors such as polarity, steric nature, solubility in solvents and concentration of monomers in the grafting solution. However, the efficiency of grafting in solutions will depend on the relative reactivity of monomers and solvent as well as the radical formation on the chains of polymer and its concentration. The free radical concentration depends mainly on the total absorbed dose. Figure 1 shows the effect of dose on the grafting yield. The grafting yield increased quickly with total dose at the beginning and then tended to level off around $10 \mathrm{kGy}$ because the free radicals increase with dose at the beginning. As the irradiation dose increases up to a certain point, the accumula- 
tion of the free radicals achieve the equilibrium at this point. Optimum grafting is achieved at a dose of $10 \mathrm{kGy}$ for all solvents. Irradiation dose of $10 \mathrm{kGy}$ was used for radiation grafting in the following study.

\subsection{Determination of Flory-Huggins interaction parameter}

The Flory-Huggins (solubility) parameter is defined as the square root of the cohesive energy density (CED) (Hildebrand and Scott 1964). The CED of a material is the increase in the internal energy per mole if all of the intermolecular forces are eliminated. The physical quantity of CED can be a useful parameter to explain, quantify or even predict the behaviour in terms of barrier properties of polymeric materials. The Flory-Huggins interaction parameter between solvent and polymer or monomer, $\chi$, is given by (Miller-Chou and Koenig 2003)

$$
\chi=V_{\mathrm{s}} \frac{\left(\delta_{1}-\delta_{2}\right)^{2}}{R T}
$$

where $\delta_{1}$ and $\delta_{2}$ are the solubility parameters of solvent, polymer or monomer, respectively, $V_{\mathrm{s}}$ the partial molar volume of the solvent and $R$ the universal gas constant. The solubility parameter values for LDPE and MMA were reported to be 7.7 and $8.7\left(\mathrm{cal} / \mathrm{cm}^{3}\right)^{1 / 2}$, respectively (Seymour and Carraher 1992). Table 1 shows the different physical properties for different solvents and the calculated $\chi$ values at room temperature.

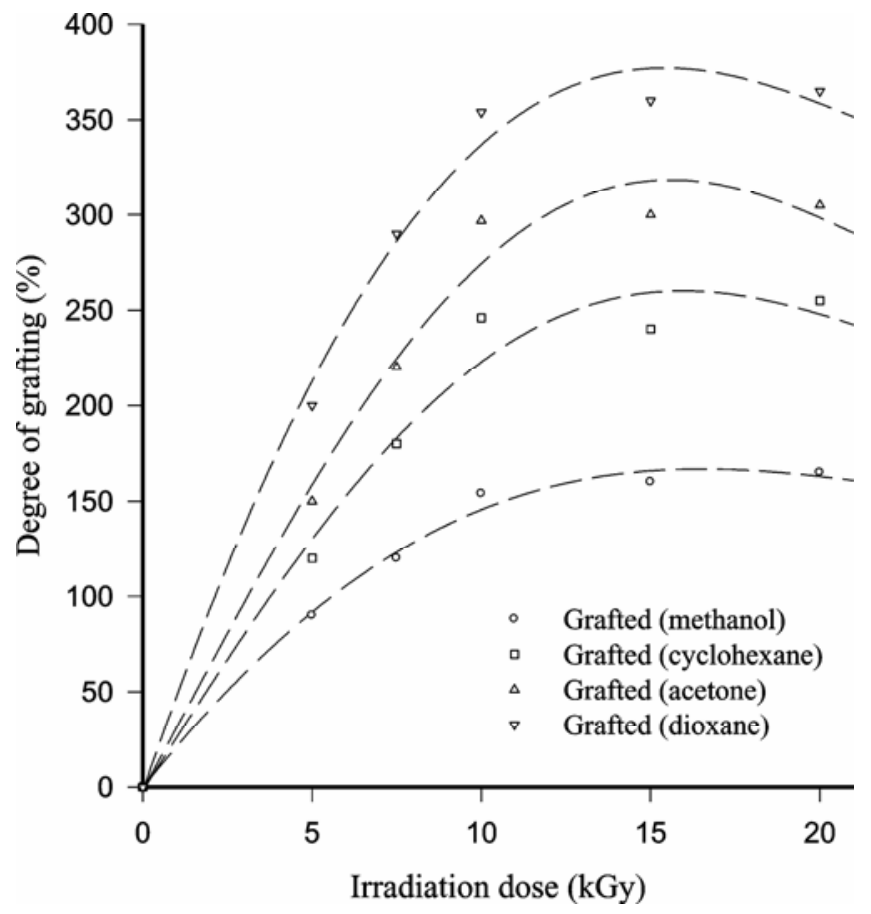

Figure 1. Effect of irradiation dose on the degree of grafting of MMA onto LDPE.

\subsection{Effect of CED of solvents on degree of grafting}

Figure 2 shows the effect of different solvents on the degree of grafting of MMA monomer onto LDPE films. All the samples had a different degree of grafting due to the used solvent. The extent of grafting, however, increases progressively when the solvent is changed from methanol to cyclohexane to acetone and to dioxane, this increase in grafting is due to the gradually increased swelling properties of the solvent (Lenka 1982). The solubility parameters, $\delta$, of the solvents should be close to the polymeric backbone so that the necessary chemical energy is produced to disrupt intermolecular cohesive forces between polymer chains and permit chain mobility.

\subsection{Structural characterization}

3.4a Scanning electron microscopy (SEM): Figure 3 shows SEM micrographs of the fracture surfaces of unirradiated, irradiated LDPE to dose of $10 \mathrm{kGy}$ of gamma radiation and grafted LDPE films in the presence of different solvents. The surfaces of both unirradiated and irradiated LDPE were smooth with no observable discontinuities. The SEM micrographs of grafted samples showed a different morphology, in which the surfaces are rough due to distribution caused by the grafted chains. Also, it can be seen that the fracture surfaces are full of white spots dispersed along the whole surfaces as shown in figure 3(c-f). This morphology depends largely on the type of solvent. From SEM micrographs of the grafted samples, it may be concluded that the solvent plays an important role on the homogeneity of grafting of MMA onto LDPE. In the case of grafting in the presence of dioxane solvent, the grafted chains tend to cover the fracture surfaces, whereas in the case of methanol the grafted chains appear as dispersed phase scattered onto LDPE surface.

3.4b X-ray diffraction (XRD): Figure 4 shows the XRD patterns of unirradiated LDPE, irradiated LDPE at $10 \mathrm{kGy}$ and LDPE graft copolymers prepared in different solvents. For unirradiated, irradiated and LDPE graft copolymer prepared in methanol, there are mainly two crystalline peaks in the XRD patterns; one in the angular range $(2 \theta)$ of $21-22 \cdot 4^{\circ}$ and another in the range $35 \cdot 4-35 \cdot 8^{\circ}$. This finding confirms the crystallinity of polyethylene, which corresponds to an orthorhombic unit cell. These peaks are well characterized in LDPE and correspond to specific crystallographic planes 110 and 200 (Chattopadhy et al 2001). There is an increase in the intensity of the peaks of the irradiated sample, which indicates an increase in the crystalline nature of the polymer. The intensity of the crystalline peaks of LDPE graft copolymers decreases with increasing degree of grafting and there is a noticeable broadening of the XRD peaks in the range $2 \theta=10-25^{\circ}$ 
Table 1. Physical properties of solvents/polymer or/MMA systems at $300 \mathrm{~K}$.

\begin{tabular}{lcccc}
\hline Solvent & $\delta\left(\mathrm{cal} / \mathrm{cm}^{3}\right)^{1 / 2}$ & Molar volume $\left(\mathrm{cm}^{3} / \mathrm{mole}\right)$ & $\chi$ (solvent-polymer) & $\chi$ (solvent-monomer) \\
\hline Methanol & $14 \cdot 5$ & 41 & $3 \cdot 18$ & $2 \cdot 31$ \\
Cyclohexane & $9 \cdot 9$ & 109 & $0 \cdot 88$ & $0 \cdot 26$ \\
Acetone & $9 \cdot 9$ & 74 & $0 \cdot 60$ & $0 \cdot 18$ \\
Dioxane & 10 & 86 & $0 \cdot 76$ & $0 \cdot 24$ \\
\hline
\end{tabular}

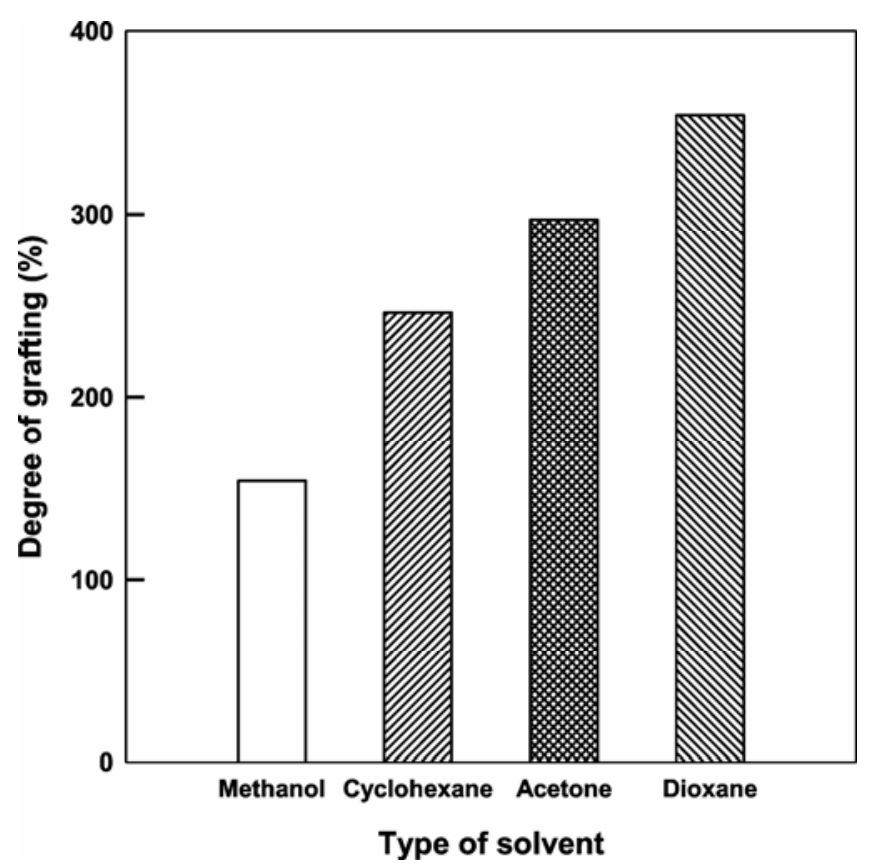

Figure 2. Effect of solvent type on the degree of grafting of MMA onto LDPE by gamma irradiation at a constant dose of $10 \mathrm{kGy}$.

for all the grafted films. These features are indicative of a reduction in the crystallinity of the LDPE with increasing degree of grafting. These XRD peaks were slightly shifted, indicating the interaction of PMMA with the polyethylene matrix. Since the XRD curves of the LDPE graft copolymers did not show any new diffraction peak, it implies the amorphous structure of PMMA components. However, the XRD peaks corresponding to the crystalline reflections for the graft copolymer appeared at identical diffraction angles as in blank LDPE. These results suggested that the inherent crystallinity of the backbone polymer was not employed by the graft copolymerization of MMA and that the grafting occurred only in the amorphous regions (Toi et al 1976; Hegazy et al 2005).

The area under the crystalline and amorphous portions was determined in arbitrary units and the degree of crystallinity $\left(x_{\mathrm{c}}\right)$ was calculated using the relation (Chattopadhy et al 2001)

$$
x_{\mathrm{c}}=\frac{I_{\mathrm{c}}}{I_{\mathrm{c}}+I_{\mathrm{a}}},
$$

where $I_{\mathrm{a}}$ and $I_{\mathrm{c}}$ are the integrated intensity corresponding to amorphous and crystalline phases, respectively. Also, the crystallite size $(P)$, interchain distance $(r)$, interplanar distance $(d)$ and distortion parameter (lattice strain) $(g)$ were calculated as follows (Alexander 1980; Chattopadhy et al 2001)

$$
\begin{aligned}
& P=\frac{k \lambda}{\beta \cos \theta}(\lambda=1.5418 \AA), \\
& r=\frac{5}{8} \frac{\lambda}{\sin \theta}, \\
& d=\frac{\lambda}{2 \sin \theta}, \\
& g=\frac{\beta}{\tan \theta},
\end{aligned}
$$

where $\beta$ is the half-height width (in radian) of the crystalline peak and $\lambda$ the wavelength of X-ray radiation and $k$ the Scherrer constant taken as 0.9 (Alexander 1980; Chattopadhy et al 2001). $P, r$ and $d$ are calculated with respect to the most intense crystalline peak at the angular range of $10 \cdot 5-10 \cdot 8^{\circ}$. Table 2 shows the different XDR parameters for all the LDPE materials. It can be seen that the percentage of crystallinity and crystallite size systematically decreased with increase in DG. Interplanar and interchain distances were marginally changed because the angle of the peak $(\theta)$ did not vary significantly, despite the variation of degree of grafting. The lattice strain decreases with irradiation while it increases with DG, which is due to the reorganization of the polymer network.

3.4c Optical properties: The law of absorption of light $I=I_{0} \exp (-\alpha X)$ was used to calculate the absorption coefficient according to the Davis and Mott (1979) model where $\alpha(\lambda)$ is the absorption coefficient which can be calculated from the optical absorption spectrum using the relation

$$
\alpha(\lambda)=2 \cdot 303 A X^{-1},
$$

where $X$ is the sample thickness, $\lambda$ the wavelength of the incident photons and $A$ is defined by $A=\log \left(I_{0} / I\right)$, 

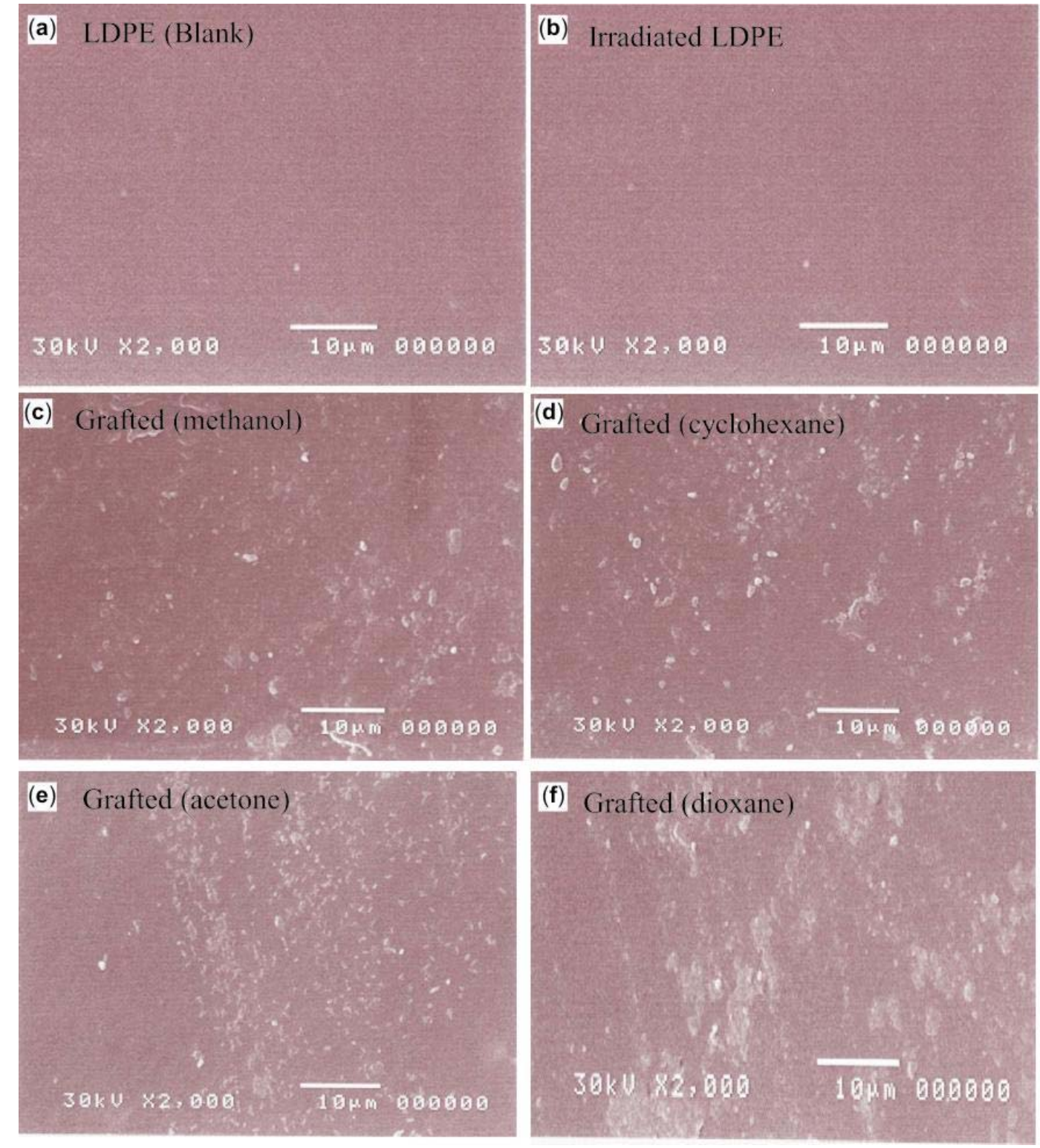

Figure 3. SEM photographs of the free surface of LDPE (test sample) and grafted with MMA, irradiated with gamma rays. Plan view: (a) un-irradiated LDPE, (b) irradiated LDPE, (c) 154\% graft, (d) $246 \%$, (e) $297 \%$ and (f) $354 \%$.

where $I_{0}$ and $I$ are the intensities of the incident and transmitted beams, respectively.

The UV/VIS spectrophotometric scans were measured in the wavelength range $200-900 \mathrm{~nm}$ for all samples. The calculated $\alpha$ for unirradiated and irradiated LDPE films is shown in figure 5a. From this figure, it is clear to see that the unirradiated LDPE has no absorption bands in the studied wavelength range. On the other hand, the spectra of irradiated films showed a shoulder band at $\sim 220 \mathrm{~nm}$. This band is attributed to the $\mathrm{C}=\mathrm{O}$ group in polymeric macromolecule. This indicates that the forbidden band width decreases with irradiation and the absorption coefficient value increases with irradiation. As shown from figure $5 \mathrm{~b}$, the grafted films showed a shoulder band shift- ing towards longer wavelength than $\mathrm{S} 2$, indicating the interaction of PMMA with the polyethylene matrix. It is obvious that the optical absorption edges are not sharply defined which characterizes the nature of samples.

The absorption edge of non-crystalline materials gives a measure of the band strengths or energy bandgap, while the main feature of the absorption edge of amorphous materials is an exponential increase of the absorption coefficient with photon energy. When the energy of the incident photon is less than the bandgap, the increase in absorption coefficient is followed with an exponential decay of density of states of the localized band into the gap (Cody 1993; Abay et al 1999). The absorption edge here is called Urbach edge $\alpha$, where the values are between 
Table 2. XRD parameters for LDPE graft copolymers

\begin{tabular}{|c|c|c|c|c|c|c|c|}
\hline Type of solvent & Peak angle $(\theta)$, degree & $(\beta)$ & $x_{\mathrm{c}}(\%)$ & $P(\AA)$ & $r(\AA)$ & $d(\AA)$ & $g(\%)$ \\
\hline Unirradiated & $10 \cdot 68$ & 0.786 & $28 \cdot 5$ & $1 \cdot 80$ & $5 \cdot 20$ & $4 \cdot 16$ & $4 \cdot 17$ \\
\hline Irradiated & $10 \cdot 68$ & $0 \cdot 684$ & $36 \cdot 7$ & $2 \cdot 06$ & $5 \cdot 20$ & $4 \cdot 16$ & $3 \cdot 63$ \\
\hline Methanol & $10 \cdot 78$ & $0 \cdot 962$ & $18 \cdot 7$ & 1.47 & $5 \cdot 15$ & $4 \cdot 12$ & $5 \cdot 05$ \\
\hline Cyclohexane & $10 \cdot 69$ & $1 \cdot 007$ & $12 \cdot 2$ & $1 \cdot 40$ & $5 \cdot 19$ & $4 \cdot 15$ & $5 \cdot 33$ \\
\hline Acetone & $10 \cdot 69$ & $1 \cdot 314$ & $11 \cdot 2$ & $1 \cdot 07$ & $5 \cdot 19$ & $4 \cdot 15$ & $6 \cdot 96$ \\
\hline Dioxane & $10 \cdot 52$ & $1 \cdot 339$ & $10 \cdot 7$ & $1 \cdot 05$ & $5 \cdot 28$ & $4 \cdot 22$ & $7 \cdot 21$ \\
\hline
\end{tabular}

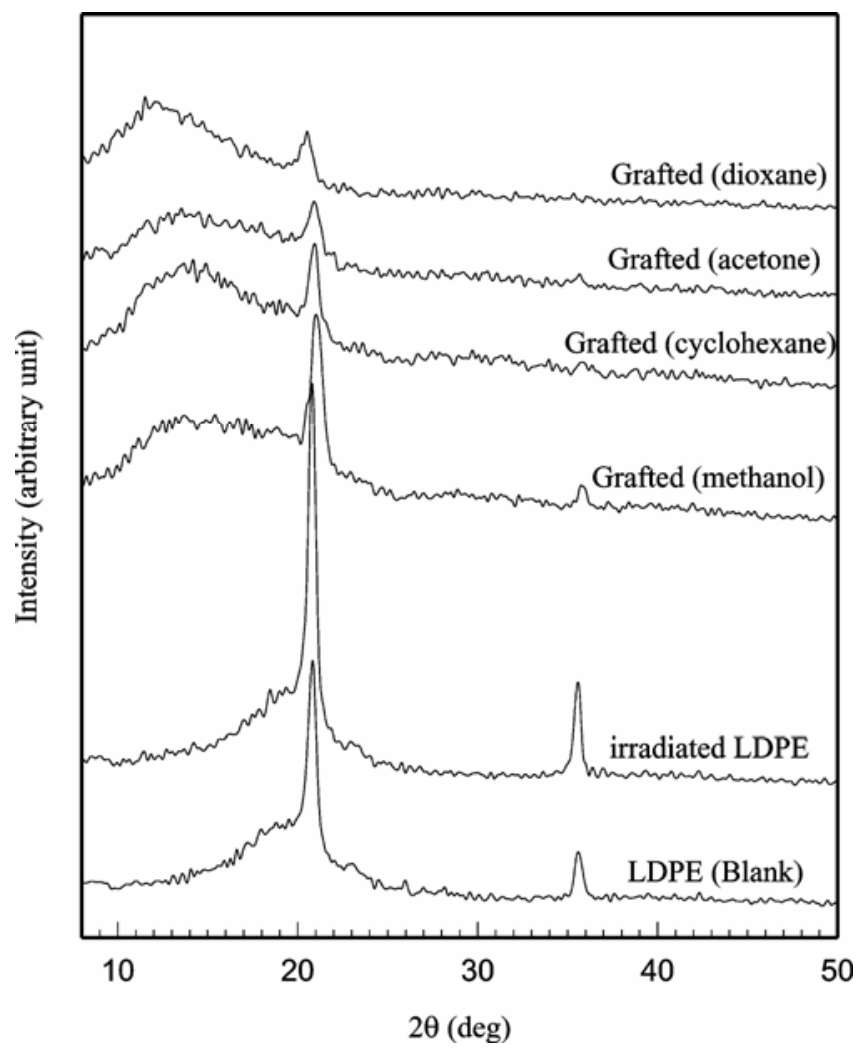

Figure 4. XRD patterns for LDPE-MMA graft copolymers prepared by gamma irradiation at a constant dose of $10 \mathrm{kGy}$.

10 and $10^{3} \mathrm{~cm}^{-1}$. The lack of crystalline long-range order in polymeric materials is associated with a tailing of density of states into normally forbidden energy (AbdelBaki et al 2006). Urbach energy characterizes the extent of the exponential tail of the absorption edge. The width of Urbach tail is an indicator of defect levels in the forbidden gap. The following relation was used to calculate the width of the Urbach tail, $E_{\mathrm{U}}$ (Urbach 1953)

$$
\alpha(v)=\beta \exp \left(\frac{h v}{E_{\mathrm{U}}}\right),
$$

where $\beta$ is a constant, $E_{\mathrm{U}}$ the Urbach energy which indicates the width of the band tails of the localized states, $h$ the Plank's constant and $(v)$ the frequency of the incident photons. The exponential absorption tails, i.e. Urbach energy, depends on temperature, thermal vibrations in the lattice, induced disorder, static disorder, strong ionic bonds, and on average photon energies. The origin of $E_{\mathrm{U}}$ is considered as thermal vibrations in the lattice (Migahed and Zidan 2006). The natural logarithm of the absorption coefficient, $\alpha(v)$, was plotted as a function of the photon energy $(E=h v)$ for LDPE films grafted with different $D G$ as in figure 6 . The values of the $E_{\mathrm{U}}$ were calculated by taking the reciprocal of the slopes of the linear portion in the lower photon energy region of these curves. The values of $E_{\mathrm{U}}$ for the studied system are calculated and listed in table 3 . The value of $E_{\mathrm{U}}$ for irradiated LDPE was found to be lower than that before irradiation while it increases upon grafting. The increase of $E_{\mathrm{U}}$ values upon grafting indicates an increase in disorder in the films.

Davis and Mott (1970) gave an expression for the absorption coefficient, $\alpha(v)$, as a function of photon energy $(h v)$ for direct and indirect optical transitions through the following equation

$$
\alpha(v)=\alpha_{0} \frac{\left(h v-E_{\mathrm{g}}^{\mathrm{opt}}\right)^{n}}{h v},
$$

where $h v$ is the energy of incident photons, $\alpha_{0}$ a constant related to the extent of the band tailing, $E_{\mathrm{g}}^{\text {opt }}$ is the value of optical energy gap between the valence band and the conduction band, and $n$ the power, which characterizes the transition process in the K-space. Specifically, $n$ is $1 / 2 ; 3 / 2 ; 2$ and 3 for transitions direct allowed, direct forbidding, indirect allowed and indirect forbidden, respectively. $\alpha_{\mathrm{o}}$ depends on the transition probability and can be assumed to be constant within the optical frequency range. The usual method for the determination of the value of $E_{\mathrm{g}}^{\mathrm{opt}}$ involves plotting $(\alpha h v)^{1 / n}$ against $(h v)$. The dependence of $(h v)^{1 / n}$ and photon energy $(h v)$ was plotted for the studied films using different values of $n$, the best fit was obtained for $n=2$. This indicates that the transition energy for electrons is indirect in K-space and interactions with lattice vibrations (phonons) take place. In other words, the minimum of the conduction band lies in a different part of K-space from the maximum of the valence band. Plots of $(\alpha h v)^{1 / 2}$ versus $(h v)$ near the absorption edge for all films produce a linear fit over a wider range of $h$ as shown in figure 7. Extrapolating the straight parts of these relations to the $h v$ axis yields the corresponding forbidden band width $\left(E_{\mathrm{g}}^{\mathrm{opt}}\right)$. The values 
of $E_{\mathrm{g}}^{\mathrm{opt}}$ for LDPE films are adapted from figure 7 and listed in table 3 . It is clear that the $\gamma$-radiation causes a shift in $E_{\mathrm{g}}^{\text {opt }}$ to a higher value. However, $E_{\mathrm{g}}^{\text {opt }}$ values decreased with grafting. This implied that the grafting leads to the appearance of the energy states induced upon radiation-induced grafting and depends on degree of grafting. Similar behaviour was observed on the spectral analysis of radiation-induced grafting of $N$-vinyl pyrrolidone (NVP) onto low density polyethylene (LDPE) films (El-Sayed et al 2003).

\subsection{Thermogravimetric analysis (TGA)}

TGA is widely used to evaluate the thermal stability of polymeric materials. Also, the initial TGA thermograms can be used to determine the activation energy of the thermal decomposition reaction. Figure 8 shows the initial TGA thermograms and the rate of reaction curves for LDPE graft copolymers prepared in different organic solvents. The TGA data corresponding to the temperatures of the maximum value of the rate of reaction $\left(T_{\max }\right)$, and the weight loss (\%) at different decomposition temperatures for virgin LDPE and grafted LDPE are listed in table 4

As can be seen from thermogram shown in figure 8, virgin LDPE film undergoes one-step degradation where
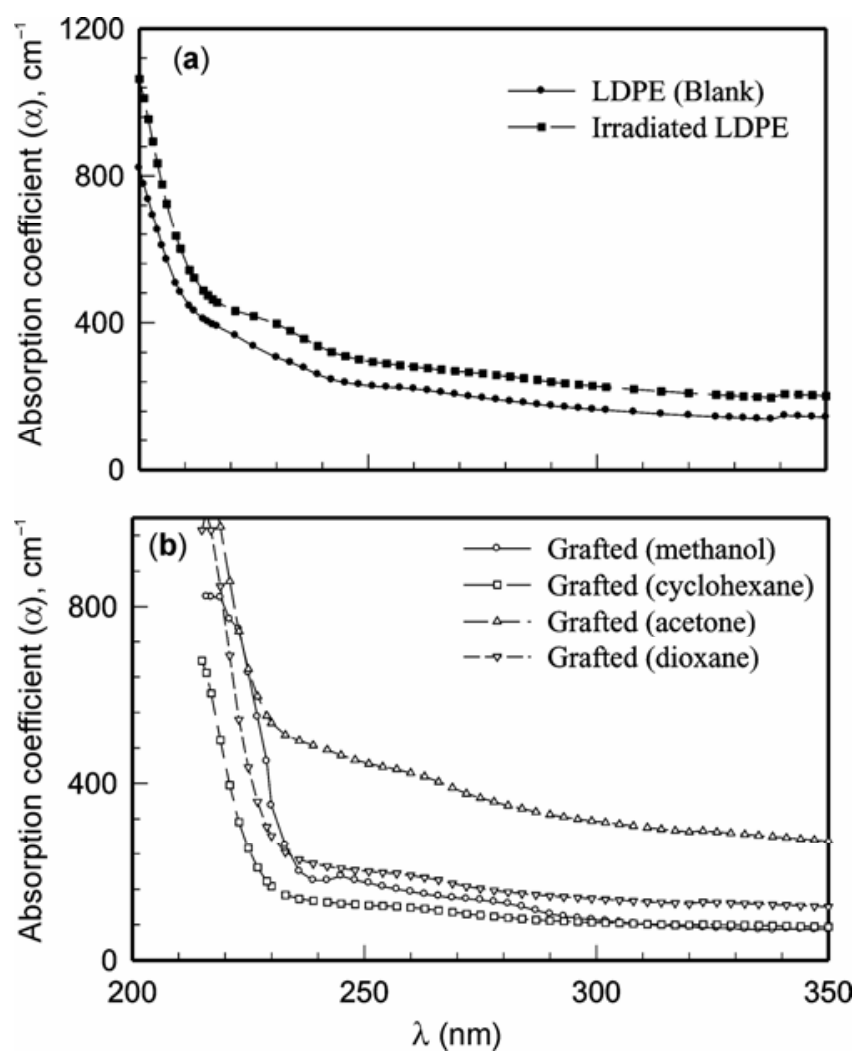

Figure 5. The absorption coefficient, $\alpha(v)$ vs wavelength of (a) LDPE, irradiated LDPE and (b) grafted LDPE. it sustains temperature up to $400^{\circ} \mathrm{C}$. The ungrafted LDPE displayed higher thermal stability than LDPE graft copolymers, regardless of the type of solvent. The thermal stability of LDPE graft copolymers above $400^{\circ} \mathrm{C}$ goes down systematically with the degree of grafting. It was also observed that with increasing degree of grafting, the thermal stability of the graft copolymers decreases than pure LDPE. This effect has already been reported by other authors about the grafting of monomers onto LDPE. Grafting of MMA onto LDPE film gives a two-step degradation pattern under the heat treatment. These trends are in agreement with reported results of radiation induced graft polymerization onto LDPE (Ferreira et al 2005). Figure 8 indicates that, when DG increases, degradation profile changes. The main degradation step of virgin LDPE shifted to low temperature in the grafted films, due to

Table 3. Optical parameters for LDPE graft copolymers.

\begin{tabular}{lccc}
\hline Sample & $\begin{array}{c}\text { Degree of } \\
\text { grafting (\%) }\end{array}$ & $E_{\mathrm{U}}(\mathrm{eV})$ & $E_{\mathrm{g}}^{\mathrm{opt}}(\mathrm{eV})$ \\
\hline LDPE (blank) & - & $0 \cdot 33$ & $3 \cdot 1$ \\
Irradiated LDPE & - & $0 \cdot 32$ & $3 \cdot 6$ \\
Grafted (methanol) & 154 & $0 \cdot 33$ & $2 \cdot 9$ \\
Grafted (cyclohexane) & 246 & $0 \cdot 33$ & $2 \cdot 6$ \\
Grafted (acetone) & 297 & $0 \cdot 36$ & $2 \cdot 3$ \\
Grafted (dioxane) & 354 & $0 \cdot 39$ & $2 \cdot 2$ \\
\hline
\end{tabular}

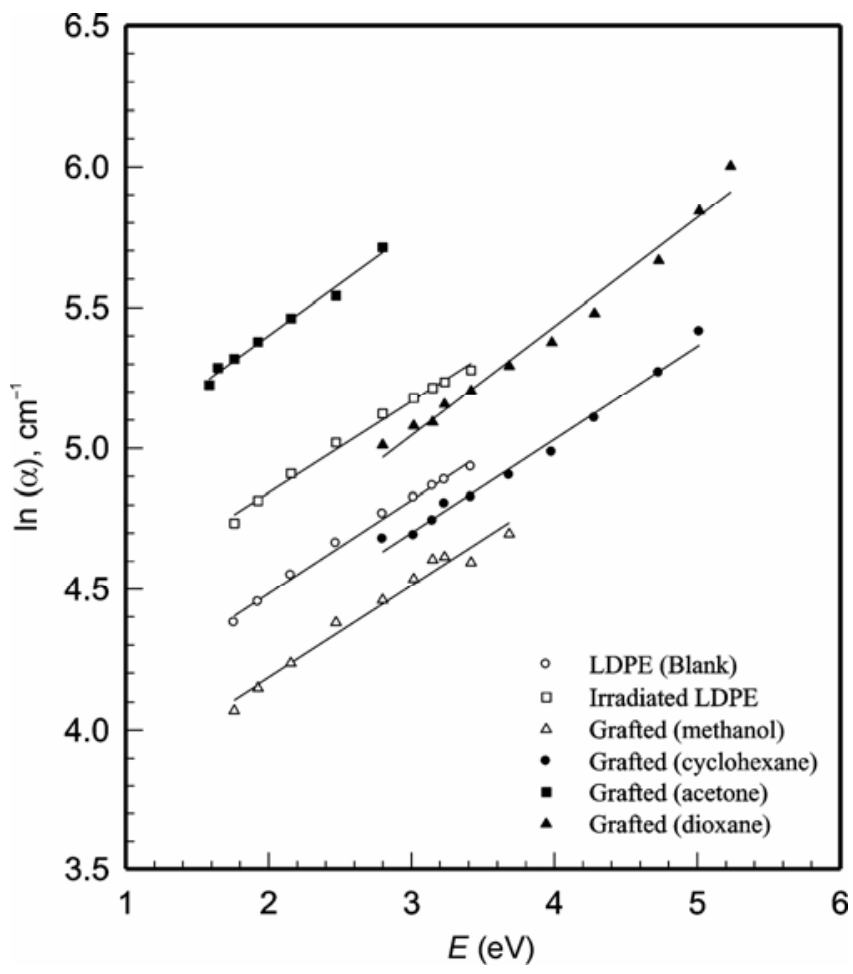

Figure 6. The logarithm of the absorption coefficient, $\ln (\alpha)$, against photon energy, $E=h v$, for LDPE, irradiated LDPE and grafted LDPE. 
Table 4. Weight loss (\%) at different decomposition temperatures and the temperatures of the maximum rate of reaction $\left(T_{\max }\right)$ for LDPE graft copolymers with MMA monomer prepared in different organic solvents.

\begin{tabular}{lcccccc}
\hline & & \multicolumn{4}{c}{ Weight loss $(\%)$} \\
\cline { 3 - 5 } Solvent & Degree of grafting (\%) & $300^{\circ} \mathrm{C}$ & $400^{\circ} \mathrm{C}$ & $450{ }^{\circ} \mathrm{C}$ & $500^{\circ} \mathrm{C}$ & $T_{\max }\left({ }^{\circ} \mathrm{C}\right)$ \\
\hline Ungrafted & - & $2 \cdot 01$ & $14 \cdot 32$ & $77 \cdot 96$ & $94 \cdot 42$ & 456 \\
Methanol & 154 & $29 \cdot 63$ & $66 \cdot 29$ & $89 \cdot 6$ & $94 \cdot 11$ & 296,397 \\
Cyclohexane & 246 & $27 \cdot 2$ & $66 \cdot 35$ & $93 \cdot 8$ & $98 \cdot 62$ & 251,391 \\
Acetone & 297 & $50 \cdot 0$ & $77 \cdot 6$ & 94.9 & 98.99 & 256,416 \\
Dioxane & 354 & $27 \cdot 88$ & $78 \cdot 1$ & $95 \cdot 85$ & $99 \cdot 53$ & 254,394 \\
\hline
\end{tabular}

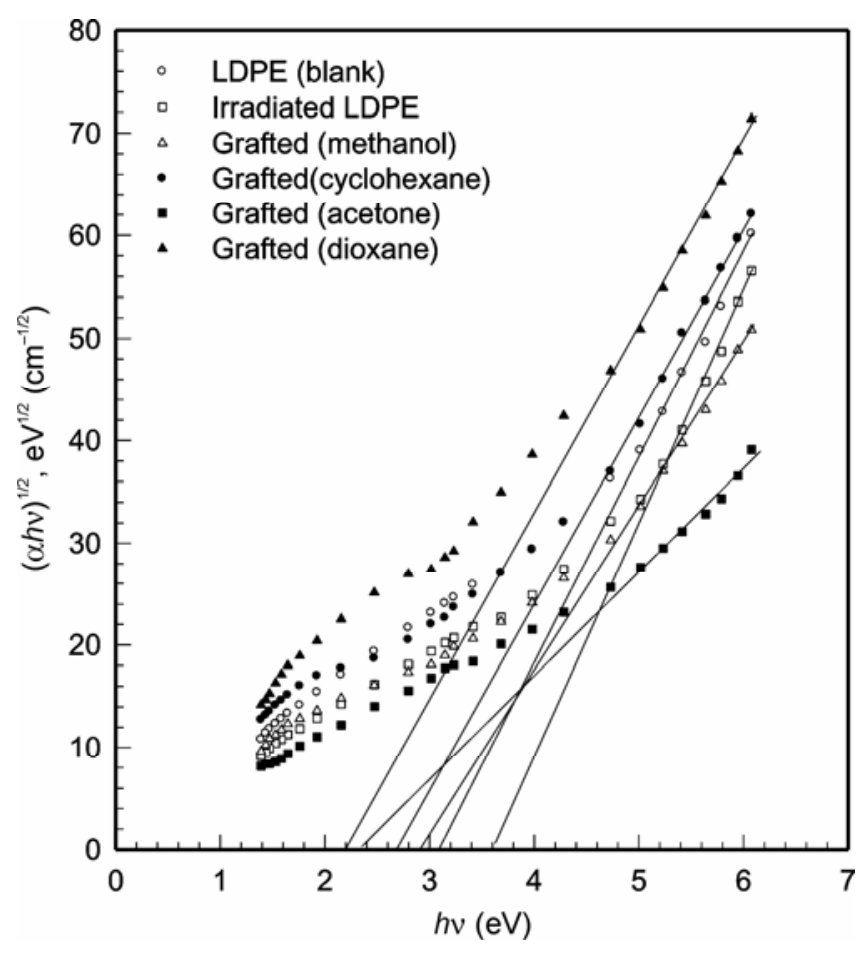

Figure 7. The plots of $(\alpha h v)^{1 / 2}$ vs $(h v)$ for all films

decrease in crystallinity and crystallite size with grafting which were explained previously.

These results suggest that MMA grafts are incompatible with LDPE matrix. It was suggested that the length of the grafted MMA grows with the increase of grafting yield, in which it is easily degraded at high temperature (Ferreira et al 2005).

The thermal stability was further confirmed by determining the energy of activation $\left(E_{\mathrm{a}}\right)$ for the thermal decomposition of LDPE before and after graft copolymerization according to Horowitz and Metzger method (Horowitz and Metzger 1963; Rao et al 2003). In this method, a plot of $\ln \left\{\ln \left[\left(W_{0}-W_{\mathrm{f}}\right) /\left(W_{t}-W_{\mathrm{f}}\right)\right]\right\}$ against $\theta$ gives a straight line with a slope of $\left(E_{\mathrm{a}} \times 10^{3}\right) / R T_{\mathrm{S}}^{2}$, in which the activation energy can be calculated from the slope. $W_{0}$ and $W_{\mathrm{f}}$ are the initial and final weights of the samples, respectively. $W_{t}$ is the weight of the sample at time, $t$ and $\theta=T-T_{\mathrm{s}} ; T$ is the temperature of the sample and $T_{\mathrm{s}}$ the

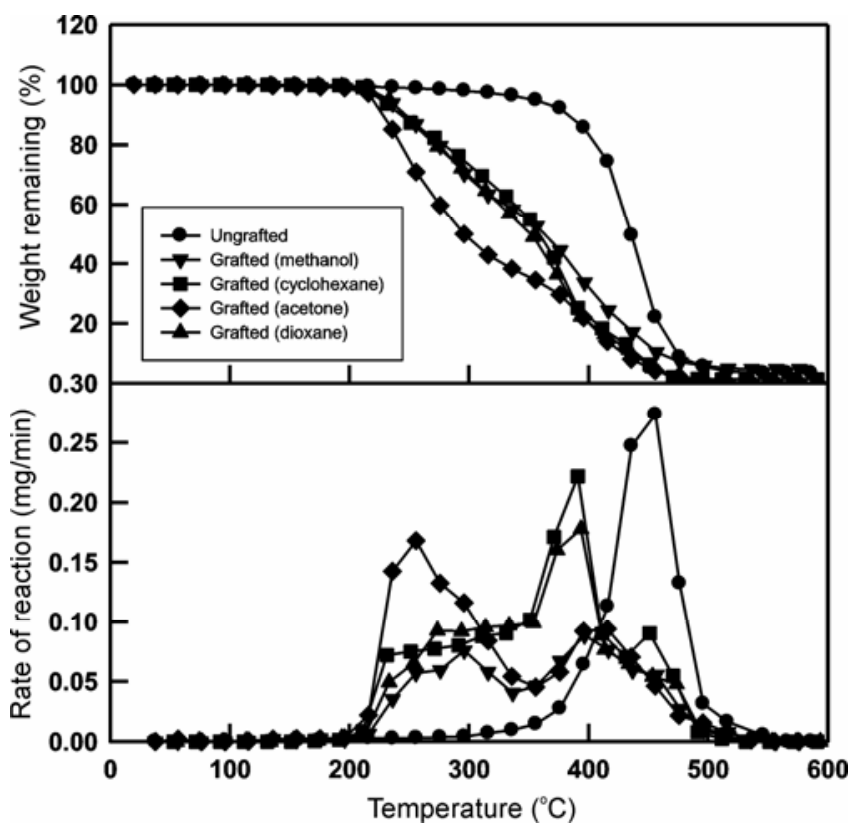

Figure 8. Initial TGA thermograms and the rate of reaction curves for LDPE graft copolymers prepared in different solvents by gamma irradiation at a constant dose of $10 \mathrm{kGy}$.

reference temperature defined as the temperature where $\left[\left(W_{\mathrm{t}}-W_{\mathrm{f}}\right) /\left(W_{0}-W_{\mathrm{f}}\right)\right]=1 / e$, where $R$ denotes the gas constant $\left(R=8.314 \mathrm{JK}^{-1} \mathrm{~mol}^{-1}\right)$. The activation energies calculated for ungrafted LDPE, graft copolymers prepared in methanol, cyclohexane, acetone and dioxane were $4.48,7.94,6.94,6.19$ and $4.5\left(10^{-16} \mathrm{eV} / \mathrm{mol}\right)$, respectively. It can be seen that the activation energy for the thermal decomposition of the grafted LDPE decreases steeply with increasing degree of grafting.

\section{Conclusions}

The changes in physical properties of LDPE films that grafted with MMA using ionizing radiation was determined and evaluated using different techniques: UV-vis, SEM, XRD and TGA. It was concluded that solvent plays an important role in the grafting process. The inherent crystallinity of the backbone polymer was not employed by the graft copolymerization of MMA and that the grafting 
occurred only in the amorphous regions. The percentage of crystallinity and crystallite size were systematically decreased with the increase in grafting degree. The value of $E_{\mathrm{U}}$ for irradiated LDPE was found to be lower than that before irradiation while it increases upon grafting, which indicates an increase in disorder in the polymer matrix. The ungrafted LDPE possessed higher thermal stability than LDPE graft copolymer, regardless of the type of solvent used. The activation energy for the thermal decomposition of the grafted LDPE decreases steeply with increasing degree of grafting due to the growth in the length of the grafted MMA with the increase of grafting yield, in which it is easily degraded at high temperature.

\section{References}

Abay B, Guder H S and Yogurtchu Y K 1999 Solid State Commun. 112489

Abdel-Baki M, El-Diasty F and Abdel Wahab F A 2006 Opt. Commun. 26165

Adem E, Avalos-Borja M, Bucio E, Burillo G, Castillon F F and Cota L 2005 Nucl. Instrum. Meth. B234 471

Alexander L E 1980 X-ray diffraction methods in polymer science (New York: Wiley Interscience)

Benson R S 2002 Nucl. Instrum. Meth. B191 752

Billmeyer F W 1984 in Textbook of polymers science (New York: John Wiley \& Sons Inc.)

Chattopadhy S, Chaki T K and Bhowmick A K $2001 \mathrm{~J}$. Appl. Polym. Sci. 811936

Chen J, Asano M, Yamaki T and Yoshida M 2005 J. Membr. Sci. 25638

Chmielewski A G, Haji-Saeid M and Ahmed S 2005 Nucl. Instrum. Meth. B236 44

Cody G D 1993 J. Non-Cryst. Solids 1413

Davis E A and Mott N F 1970 Philos. Mag. 22903

El-Sayed S M, Arnaouty M B and Fayek S A 2003 Polym. Test. 2217

Ferreira L M, Falcao A N and Gil M H 2005 Nucl. Instrum. Meth. B236 513
Hegazy E A, Kamal H, Abdel Geleel M and Abdel Maksoud A 2005 J. Appl. Polym. Sci. 95936

Hildebrand J H and Scott R L 1964 The solubility of nonelectrolytes (New York: Dover Publications) 3rd ed.

Horowitz H H and Metzger G 1963 Anal. Chem. 351464

Inami T, Kobiyama M, Okuda S, Maeta H and Ohtsuka H 1999 Nanostruct. Mater. 12657

Jastrzebski Z D 1977 The nature and properties of engineering materials (New York: John Wiley and Sons)

Jiang H G, Ruhle M and Lavernia E J 1999 J. Mater. Res. 14 549

Klug H P and Alexander L E 1974 X-ray diffraction procedures for polycrystalline and amorphous materials (New York: John Wiley \& Sons) 2nd ed.

Lenka S 1982 J. Appl. Polym. Sci. 271417

Malow T R and Koch C C 1997 Acta Mater. 452177

Migahed M D and Zidan H M 2006 Curr. Appl. Phys. 691

Miller-Chou B A and Koenig J L 2003 Prog. Polym. Sci. 28 1223

Mott N F and Davis E A 1979 Electronic processes in noncrystalline materials (Oxford: Oxford University Press) 1st ed.

Qian L H, Wang S C, Zhao Y H and Lu K 2002 Acta Mater. 50 3425

Rajindar S 1998 Chemtech 2833

Rao P S, Subrahmanya S and Sathyanarayana D N 2003 Synth. Metals 139397

Saq'an S, Ramadin Y, Ahmad M, Zihlif A, PopovskaPavlovska F and Trajkovska A 2001 Polym. Test. 20919

Seymour R B and Carraher C E Jr 1992 Polymer chemistry: An introduction (New York: Marcel Dekker) 3rd ed.

Tian H H and Atzmon M 1999 Philos. Mag. A79 1769

Toi K, Igarashi K and Tokuda T 1976 J. Appl. Polym. Sci. 20 703

Ungar T T 2003 Adv. Eng. Mater. 5323

Urbach F 1953 Phys. Rev. 921324

Utracki L A 1990 Polymer alloys and blends (Munich: Hanser Publishers)

Warren B E and Averbach B L 1950 J. Appl. Phys. 21595

Zhao Y H, Sheng H W and Lu K 2001 Acta Mater. 49 365 